\title{
A Review on Efficacy of Sensors in Capturing Biophysical Measures and its Application in the Field of Physical Therapy and Rehabilitation
}

\author{
Md Abu Shaphe', Rashid Ali Beg ${ }^{2}$, Mudasir N Shah ${ }^{3}$, Aksh Chahal ${ }^{4}$, Amr S Shalaby \\ ${ }^{1}$ Associate Professor Physical Therapy Jazan University, Saudi Arabia, ${ }^{2}$ Lecturer Physical Therapy Jazan \\ University, Saudi Arabia, ${ }^{3}$ Lecturer Physical Therapy Jazan University, Saudi Arabia, ${ }^{4}$ Professor, MM Institute \\ of Physiotherapy and Rehabilitation, Maharishi Markandeshwar (Deemed to be University), Mullana, Ambala, \\ Haryana, India, ${ }^{5}$ Lecturer, Basic Science, Physical Therapy, Cairo University, Egypt
}

\begin{abstract}
Background: Use of sensors for assessment and treating orthopedic and neurological conditions in physical therapy and rehabilitation is in practice since last 2 decades. Its efficiency has been promising with advancements in research and clinical practice.

Objective: The aim of the present review is to synthesize and evaluate studies which have performed a role to test efficacy of sensors following orthopedic and neurological conditions for research and clinical practice in physical therapy and rehabilitation.

Data Source: Are view of articles identified from high impact journal from Springer, Hindawi, MDPI, PLOSone, De Gruyter was performed by both researchers.

Study Eligibility Criteria: Articles published only in English between January, 2010 and December, 2019 were included which investigated role of sensors in physical therapy and rehabilitation.

Study Appraisal and Synthesis Method: Studies were grouped as wearable, bio-mechanical pressure, motion capture sensing technology and stretch sensors.

Results: From the 17 articles identified, 10 papers were involved in the review which was deemed as being of high quality.

Conclusion: Sensor technology is a full-fledged and rapidly growing field with imperative focus and noteworthy role in assessment and treatment for upper and lower limbs insufficiencies, spinal curve corrections in numerous orthopedic and neurological conditions. Research over the past 10 years grandly focused on the use of sensors in physical therapy and rehabilitation.
\end{abstract}

Keywords: Sensor, rehabilitation, physical therapy, orthopedic, neurology.

\section{Introduction}

In the modern world rehabilitation has grown to

\section{Corresponding Author:}

\section{Aksh Chahal}

Professor, MM Institute of Physiotherapy and

Rehabilitation, Maharishi Markandeshwar (Deemed to be University), Mullana, Ambala, Haryana, India e-mail:drakshchahal@gmail.com its appreciable level of invention and deployment. This still has not led to stopping in induction of new and advanced inputs in different domains of healthcare, bio-engineering, sports and entertainment to work collectively towards betterment of mankind ${ }^{[1]}$. One of the widely developed measures being used in assessment and treatment in physical therapy and rehabilitation isthe use of 'Sensors' ${ }^{[2]}$. Sensors installed in equipments or wearable component have been used in rehabilitation of orthopedic and neurological for performing functions 
of developing motivation while commencing of rehabilitation programs ${ }^{[2,3]}$. Monitoring correctness and irregularities in patterns different from normalprototype, identification of setbacks and unwarranted behavior ${ }^{[1,4]}$. Regular association between health information being transferred to health care professionals as exact and confirmative values ${ }^{[5]}$. Data stored in the sensors to be transferred and expanded to provide visual and auditory feedback to subjects ${ }^{[6-7]}$.Sensors are being used while performing exercise, in corsets as wearable sensororthosis, walking aids, attached to elastic bands for shoulder, knee and hip rehabilitation along with specialized ergonomic equipments ${ }^{[8-10]}$. The two mainly used equipment involving sensors in physical therapy and rehabilitation are '3D motion capture systems' and 'depth camera system' such as Microsoft Kinect in assessing the application of sensors in neurological conditions of Parkinson's disease, Cerebral Palsy and patients with Multiple Sclerosis ${ }^{[1]}$. The researchers found the technique to be low cost and acceptably reliable but could be used for upper and lower limb rehabilitation training and monitoring balance and increasing the range of movement for both, upper and lower limbs. Both systems pose their respective advantages over each other with even their setbacks. 3D motion capture technology is expensive wherein the skin mounted sensors have a tendency to hinder the movement but provide an advantage of providing accuracy in collection of data ${ }^{[11,12]}$. But after collection of data, processing the data with recommendations to the subject by a therapist requires deep knowledge regarding the functioning, calibration and interpretation with subject's findings. Hence, this system is used by priority only in research setting than used for clinical practice ${ }^{[13]}$. However, depth cameras systems like the Microsoft Kinect offer an ease in use wherein data can be easily calibrated and interpretive ${ }^{[3,14]}$. But it poses a disadvantage of slight lower in accuracy than 3D motion capture technologies ${ }^{[14]}$. Following to easy portability and cost efficiency this system is grasped for research, commercial and clinical benefits. The common disadvantage seen with use of depth camera systems is the crossing over seen in body segments during action,in addition with inappropriate lightening effects and hindrance caused due to movement of people and restrictions offered from self-clothing ${ }^{[15]}$. Lastly, this system requires significant empty and large area to be used in practice which is usually a constraint in small research laboratories and clinical settings. This systematic review would organize and discuss use of sensors in the field of physical therapy and rehabilitation.

\section{Method}

\section{Literature source strategy and study selection}

Process: In the present review, authors present with a systematic review for using sensors in physical therapy and rehabilitation as per the PRISMA (Preferred Reporting Items for Systematic Reviews and MetaAnalyses) statement ${ }^{[16]}$.

A literature search was conducted with $\mathrm{MeSH}$ (Medical Subject Heading) terms, abstract keywords and titles along with their synonyms and spelling variations in combinations for all database. The general search strategy including the search terms used in the review are mentioned in table 1 .

Table 1. Literature search strategy

\begin{tabular}{|l|l|}
\hline \multirow{2}{*}{ Physical therapy } & $\begin{array}{l}\text { 'sensor', 'Sensors', 'Bio-feedback sensor', } \\
\text { 'Wearable sensor', 'Stretch sensor', }\end{array}$ \\
\hline Rehabilitation & $\begin{array}{l}\text { 'Sensor', 'Sensors', 'Bio-feedback sensor', } \\
\text { 'Wearable sensor', 'Stretch Sensor', } \\
\text { 'Sensing' }\end{array}$ \\
\hline
\end{tabular}

Reputed database were selected for gathering articles from high impact journal of Springer, Hindawi, MDPI, PLOSone, De Gruyter. Articles published between January, 2013 to December, 2019, analyzing the latest and in action utilization of sensors in physical therapy and rehabilitation were only searched and considered for the present review. While selecting articles to be considered in the review, conference proceedings' were also considered to be instituted, but no proceedings were attained by the authors. Search did not limit an article based on publication date during the tenure. Reference list of the included articles was also searched for further prioritizing selections. Due to prove efficacy of sensors in physical therapy and rehabilitation, grey literature was not searched, rather only peer-reviewed articles were considered for the present review. The article selection process consisted of the following steps on the PRISMA guidelines which have been shown in Figure 1:

\begin{tabular}{|l|l|}
\hline The records recognized after searching database & $(\mathrm{n}=17)$ \\
\hline $\begin{array}{l}\text { Articles removed after noticing duplication of title } \\
\text { and or abstract }\end{array}$ & $(\mathrm{n}=10)$ \\
\hline $\begin{array}{l}\text { Full text articles screened based on inclusion and } \\
\text { exclusion criteria }\end{array}$ & $(\mathrm{n}=10)$ \\
\hline Number of articles finally included in the review & $(\mathrm{n}=10)$ \\
\hline
\end{tabular}

Inclusion and Exclusion Criteria: The present review emphasize on functionality of sensors in physical 
therapy and rehabilitation. Inclusion criteria for the articles were the instrument, mode, purpose and inference obtained following sensor use. Articles only with the concerned information were included to maintain homogeneity of information procured from articles for a uniform and disciplined review among all articles. Any article published before 2010 was excluded. This act was performed as the authors installed greater emphasis on articles published only in recent years which would render latest developments in sensor technology and their benefits. Articles published other than English were not included in the review. Titles and abstracts of the identified articles were read keeping in mind both the inclusion and exclusion criteria's. Articles fulfilling both the criteria only were read in full text to extract desired information.

Data Extraction Process: The work of data extraction was completed by both authors where they discussed all discrepancies in finding articles from various database with outline and in-depth understanding of the articles for common interest. To maintain compliance, a standardized data extraction form was used in the review where in the study design; types of sensors used in physical therapy and rehabilitation used were ascertained. The review were later divided in 4 types of sensors; wearable sensors, bio-mechanical pressure sensors, 3D motion capture sensors and stretch sensors for practicality in research and clinical settings.

Assessment of Study Quality: Both authors evaluated the quality of articles retrieved from database which were deemed eligible to be included in the present review. All articles were verified to contain content expected to be present in an article to be considered to perform a review to be published in journals of high repute as journals with high impact factor prefer articles written following guidelines as per the universally accepted protocols only. Hands on self-administered protocols are rarely given place in journals of prestigious honor. Keeping this view in mind, strict adherence was followed while performing the present review.

\section{Results}

Database Search and Paper Lists: A detailed overview of results at different stages following PRISMA guidelines have been shown in Figure 1. From the 17 articles that were identified in the search categories, 10 papers of high repute with vast content of information was only included in the present review following the selection criteria. While searching all articles it was noticed that the not included 7 article contained the inclusion criteria to be selected in the study but in addition even contained parameters which were set as exclusion criteria making the authors of the review to finally exclude them to prevent biasing and interpretation of incomplete and irrelevant information. After reading the extracted information, sorting and documentation of information has been presented.

\section{Discussion}

Sensors with their ability towards precision and accuracy are widely used for assessment, progression and rehabilitation in physical therapy and rehabilitation in the domain of orthopedics and neurology producing promising results when used among age groups from childhood to geriatric population. Studies were evaluated based on their type of sensor being used, instrument used for installing the same, purpose of the same and results delivering on implementation of the sensors. Studies presented with positive implications of sensors in subjects by enhancing the overall physical and mental status of the subject leading to an improvement in the overall quality of life. Studies included in the present review presented a wide option of sensors like wearable sensors, bio-mechanical pressure sensors, motion capture sensing technology and stretch sensors delivering services to yield excellent and observable positive results in time to time recovery and enhancement of functions affecting the overall physical, mental and psychological mindset of the subjects'. Researchers emphasized if possible the use of sensors to start right from the assessment phase which will help significantly in predicting the realistic expectations to be set according to individual subject's physical and mental status. Faster and focuses assessment brings effective and timely rehabilitative effects leading to reduction in unwanted cost on the patients and country's economy. The researchers also noticed that few sensor technologies are expensive, but on the hand cost efficient modes are also available in the market to cater all sections of the society yielding promising results without compromising with accuracy and overall quality of the product. Application of sensors in gadgets such as corsets, mobile phones and walking aids have been be promoted both at research and clinical level to train patient with pathologies, disorders to move forward to faster recovery. Elderly population could be started with training with markers followed by application of sensors in quadripod, tripod and cane respectively to improve balance and reduce the 
risk of fall which have been proved in researchers from all over the world to be a major source of injury seen among elderly population. A progression from a wider base of support to a narrower one with using sensors imparts visual feedback from the equipment itself while testing and training used in conjugation with the sensors and in addition from the auditor stimuli received from the equipment and commands from the therapist to promote variability and extensibility in enhancement of movement and functions while performing all major to perform activities of daily living thus, bringing back the subject to his optimum level for self-independence in daily life.

Review Limitations: Despite the strength of the present review, it is mandatory for a researcher to consider limitations while result interpretation. Studies other than English language were not included in the review, which can influence the outcomes of present analysis as certain database comprise of journals in context to language other than English. So, due to this limitation, articles published in those databases might be contained with latest and valuable information to increase the potency of the current review. Both authors were actively involved in data extraction as there is possibility of wrong exclusion of an article to be included in the present review. This article is in compliance with PRISMA guidelines, but the protocol of the present review did not gothrough a registration prior to its completion. Articles before 2010 were not included in the present review along with articles were not selected only from a particular database.

Practical Implications: Yurtman A et al, Hondori $\mathrm{HM}$ et al, Postolache $\mathrm{O}$ etal, Rathleff MS et al, Vallati $\mathrm{C}$ et al, Spilz A et al and Kongcharoen $\mathrm{J}$ et al, studied the role of sensors specifically in condition dealt by physical therapist on daily basis using equipments in routine protocols for assessment and rehabilitation. The researchers proved sensor technology when used in physical therapy as cost effective with few delivering accurate and some low level of accuracy in results but still could be used assessing with their added advantages' over the expensive ones and bulky to be installed in research and clinical practice. Sensor technique was found to be effective in storing data and later on retractions followed by interpretations in strength training techniques, early correction of spinal curves, increasing the range of motion of upper and lower limbs by focusing on individual or cumulative joints in a trajectory and instigating adjustments to be made at home for activities such as walking, bathing, eating etc to attain independence in activities of daily living. Parameters like stride and step length, step length, cadence, acceleration and deceleration during swing phase and ground reaction forces acting on individual joints during stance along with foot placement deviations and anomalies could be assessed and measured by using sensors in clinical and research settings. Unnikrishnan $\mathrm{R}$ et al found use of video games in combination with sensors too were found to be effective to promote exercise on daily basis with bodily movement seem an increase in range of motion and muscle strength when used for treatment of upper limbs. Kristof $M$ et al suggested sensors when installed in a mechanical pressure named equipment Pressure $\mathrm{X}$ promote significant reduction in scoliotic curves wherein the major corrections were seen in thoracic curves, followed by thoraco-lumbar and less correction were seen in lumbar curves which they attributed due to large size of vertebrae which once fused during spinal deformities are driven by less chance to correct to greater modifications. Kent $\mathrm{P}$ et al emphasized assessing the Cognitive Functional Therapy by using sensor feedback in treating subjects with chronic low back pain. The researchers found it to be an effective technique for the same and suggested the same protocol to be carried out in conducting Randomized Controlled Trials with substantial subjects in different demographic locations with gender variation and varied age groups ${ }^{17}$.

\section{Conclusion}

Research in the past 10 years has involved use of sensor both in physical therapy and rehabilitation for orthopedic and neurological pathologies and conditions. Sensor technology has been a boon in rehabilitation helping millions of people from varied age groups. The use of sensor use in assessment and rehabilitation enables both therapist and patient to isolate the validated improvement during interventions and identify from a small to major deviation during the treatment. There exist a vast number of considerations of sensor use in conditions specific to individual joints which are to be characterized either in orthopedic and neurological domain to be studied individually to obtain data for further joint wise reviews estimating, use of sensor role concerning the efficacy of sensors. Such studies if promoted will be vital in developing advanced and cost effective assessment and treatment strategies.

Ethical Clearance: Not Applicable 
Source of Funding: Self

Conflict of Interest: Nil

\section{References}

1. Yurtman A, Barshan B. Detection and Evaluation of Physical Therapy Exercise by Dynamic Warping Using Wearable Motion Sensor Units. Information Sciences and Systems. 2013: 305-314.

2. Kristof M, Hudak R, Takacova A, Zivcak J, Fialka L, Takac R. Contact Pressure Measurement in Trunk Orthoses. IEEE International Joint Conferences on Computational Cybernetics and Technical Informatics, May 27-29, Timisora, Romania. 2010: 175-179.

3. Hondori HM, Khademi M. A Review on Technical and Clinical Impact of Microsoft Kinect on Physical Therapy and Rehabilitation. Journal of MedicalEngineering.2014; ID 846514:1-16

4. Postolache O. IoT for Healthcare: Smart Physiotherapy. 22 $2^{\text {nd }}$ IMEKO TC4 International Symposium and 20th International Workshop on ADC Modelling and Testing, IASI, Romania, September, 14-15. 2017: 559-560.

5. Vallati C, Virdis A, Gesi M, Carbonaro N, Tongnetti A. e Physio: A Wearables-Enabled Platform for the Remote Management of Musculoskeletal Diseases. Sensors. 2019; 19(2):1-18.

6. Rathleff MS, Thorborg K, Bandholm T. Concentric and Eccentric Time-Under-Tension during Strengthening Exercise:Validity and reliability of Stretch-Sensor Recordings from and Elastic exercise-Band. PLoS One.e68172. 2013; 8(6):1-9.

7. Spilz A, Engleder T, Munz M, Karge $M$. Development of a smart fabric force-sensing glove for physiotherapeutic Applications. Current Directions in Biomedical Engineering. 2019; 5(1): 513-516.

8. Kent P, Sullivan PO,Smith A,Haines T,Campbell $A$ et al. RESTORE-Cognitive functional therapy with or without movement sensor biofeedback versus usual care for chronic, disabling low back pain:study protocol for a randomized controlled trial. BMJ Open.2019;9:e031133.
9. Unnikrishnan R, Moawad K, Bhavani RR. A Physiotherapy Toolkit using Video games and Motion Tracking Technologies. IEEE.2013;90-95.

10. Kongcharoen J, Pruitikanee S, Puttinaovarat S, Tubtiang Y, Chankeaw P. Gamification Smartphone Application for Leg Physical Therapy. IJOE. 2019; 15(8):31-41.

11. Ahmadi A, Mitchell E, Destelle F, Gowing M, O'Connor NE, Richter C. Automatic activity classification and movement assessment during a sports training session using wearable sensors. In: Proc $11^{\text {th }}$ Int Conf Wearable Implant Body Sens Networks, BSN.2014:98-103.

12. Bonnechere B, Jansen B, Salvia P, Bouzahouene H, Omelina L, Moiseev F. Validity and reliability of the Kinect within functional assessment activities: comparison with standard stereophotogrammetry. Gait Posture.2014;39:593-8.

13. Bonnet V, Mazza C, Fraisse P, Cappozzo A.An optimization algorithm for joint mechanics estimate using inertial measurementunit data during a squat task. Proc Annu Int Conf IEEE Eng Med Biol Soc.2011:3488-91.

14. O'Reilly M, Caulfield B, Ward T, Johnston W, Doherty C. Wearable Inertial Sensor Systems for Lower Limb Exercise Detection and Evaluation: A systematic Review. Sports Med.2018;48 (5):12211246.

15. Arulampalam J, Pierrepont J, Kark L. Markerless motion capture: validity of Microsoft Kinect cameras and ipisoft. Gait Posture.2018;42:S76.

16. Moher D, Liberati A, Tetzlaff J, Altman DG. Preferred reporting items for systematic reviews and meta-analysis: the PRISMA statement.BMJ. 2009; 339: b2535.

17. Shaphe A, Shalla I. H, Al Baradie R. S, Qasheesh M. Efficacy of Closed Loop Feedback System with Augmented Virtual Reality Visual Cues Training on Gait and Functional Performance in Stroke Patients. Biosc. Biotech. Res. Comm. 2018; 11(1).70-75. Available from: https://bit.ly/2YD7zUk 